\title{
Amnioinfusión en embarazos de pre término extremo con rotura prematura de membranas - resultados durante el embarazo, maternos y neonatales (AMIPROM study): Estudio piloto clínico, controlado randomizado (1)
}

\author{
Roberts D, Vause S, Martin W, Green P, Walkinshaw S, Bricker L, Beardsmore C, Shaw \\ $N$, McKay A, Skotny G, Williamson P, Alfirevic Z. Amnioinfusion in very early preterm pre- \\ mature rupture of membranes - pregnancy, neonatal and maternal outcomes in the AMI- \\ PROM randomised controlled pilot study. Ultrasound Obstet Gynecol 2013. doi: 10.1002/ \\ uog. 13258. \\ Análisis crítico: Fernando Ferrer Márquez ${ }^{1}$, Claudio Vera PG, MSc ${ }^{1-2}$, Jorge A. Carvajal, $\mathrm{PhD}^{1}$ \\ ${ }^{1}$ Unidad de Medicina Materno-Fetal, División de Obstetricia y Ginecología; ${ }^{2}$ Unidad de Medicina Basada en Evidencia. \\ Escuela de Medicina. Facultad de Medicina. Pontificia Universidad Católica de Chile
}

\section{RESUMEN (1)}

Antecedentes: La sobrevida fetal está severamente comprometida cuando las membranas se rompen entre las 16 y 24 semanas de embarazo. Un volumen reducido de líquido amniótico se relaciona con pobre desarrollo pulmonar mientras que un volumen apropiado de líquido amniótico lleva a mejor resultado perinatal. Restaurar el volumen de líquido amniótico mediante amnioinfusión guiada por ultrasonido puede ser de beneficio en mejorar el resultado perinatal y de largo plazo en niños de embarazos con esta condición. Objetivo: Evaluar los resultados a corto y largo plazo de las pacientes con rotura prematura de membranas muy precoz aleatorizados a amnioinfusión seriada o manejo expectante; recolectar datos para desarrollar un estudio clínico más grande y definitivo. Diseño: Estudio prospectivo, no ciego, aleatorizado y controlado, con randomización estratificada para los embarazos con rotura prematura de membranas entre las $16+0$ y 19+6 semanas de gestación y $20+0$ y $23+6$ semanas de gestación, para reducir al mínimo el riesgo de desequilibrio en la distribución de las edades gestacionales entre ambos grupos. Se utilizó el análisis por intención de tratar. Escenario: Se realizó en las unidades de Medicina Materno Fetal de cuatro hospitales en el Reino Unido (Liverpool Women's NHS Trust, St. Mary's Hospital Manchester, Birmingham Women's NHS Foundation Trust, Wirral University Hospitals Trust). Participantes: Mujeres embarazadas con rotura prematura pretérmino de membranas confirmada entre las $16+0$ y $24+6$ semanas de gestación. Se excluyeron las mujeres con embarazo múltiple, anomalías fetales o indicación de interrupción inmediata del embarazo. Intervención: Las pacientes fueron asignadas aleatoriamente a amnioinfusión transabdominal semanal seriada si el bolsillo mayor de líquido amniótico era $<2 \mathrm{~cm}$ o a manejo expectante hasta las 37 semanas de gestación. Resultados a medir: Se midieron resultado a corto plazo de las madres, embarazo y neonato, y resultados a largo plazo de los niños. La morbilidad respiratoria a largo plazo fue evaluado mediante cuestionarios validados a los 6, 12 y 18 meses de edad y las pruebas de función pulmonar alrededor de los 12 meses de edad. El neurodesarrollo fue evaluado mediante la Escala de Bayley de Desarrollo Infantil II a los dos años de edad corregida. Resultados: 58 embarazadas fue- 
ron randomizadas. Dos recién nacidos fueron excluidos del análisis debido a la interrupción del embarazo por anomalía letal dejando 56 participantes (28 en la serie de amnioinfusión y 28 en manejo expectante), reclutados entre 2002 y 2009. No hubo diferencia significativa en la mortalidad perinatal (19/28 vs 19/28; RR 1,0, IC 95\% 0,70-1,43), morbilidad materna o neonatal. La posibilidad total de sobrevivir sin patología respiratoria a largo plazo o con trastornos del neurodesarrollo fue $4 / 56(7,1 \%)$; $4 / 28(14,3 \%)$ en el grupo de amnioinfusión y 0/28 en el grupo de manejo expectante (RR 9,0; IC95\% 0,51-159,7). Conclusiones: Este estudio piloto no encontró diferencias significativas en los resultados maternos, perinatales o del embarazo. El estudio no fue diseñado para mostrar una diferencia entre los grupos y el número de supervivientes fue demasiado pequeño para extraer conclusiones acerca de los resultados a largo plazo. Señaliza sin embargo, que se necesita un estudio más grande para evaluar la amnioinfusion en mejorar la salud de los sobrevivientes. El estudio pilotó sugiere que con el financiamiento apropiado, este estudio es posible.

\section{ANÁLISIS DE LA INVESTIGACIÓN}

\section{A. Relevancia clínica de la investigación}

La rotura prematura de pretérmino de membranas ovulares durante el embarazo (RPPM) corresponde a la causa identificable más frecuente de parto prematuro y es una gran causa de morbilidad y mortalidad perinatal. La disminución de líquido amniótico (oligohidroamnios) secundaria a RPPM es uno de los parámetros más importantes de mortalidad perinatal en RPPM precoz, ya que jugaría un rol crítico en la aparición de hipoplasia pulmonar con una incidencia de un $5 \%$ a $62 \%$. El oligohidroamnios también está asociado a un alto riesgo de desarrollar infecciones intrauterinas (corioamnionitis), infección neonatal, compresión de cordón umbilical, alteraciones neurológicas, deformidad postural y parto prematuro. Los embarazos con RPPM y OHA (bolsillo de líquido amniótico menor a $2 \mathrm{~cm}$ ) presentan alto riesgo de un pobre resultado perinatal y alteraciones neurológicas a largo plazo, mientras que los pacientes con presencia de un bolsillo mayor a $2 \mathrm{~cm}$, luego de amnioinfusión o manejo expectante, tienen significativamente mejor resultado perinatal (73-92\% de sobrevida) y tasas bajas de hipoplasia pulmonar (2). Considerando estos antecedentes parece importante reducir el número de complicaciones asociadas a la presencia de líquido amniótico disminuido en las pacientes con RPPM precoz. Existen estudios previos (ob- servacionales) que sugieren que la amnioinfusión trans-abdominal puede mejorar significativamente el pronóstico perinatal en embarazos con RPPM precoz entre las 16 y 26 semanas de gestación. Un reciente meta-análisis realizado sugiere que la amnioinfusión seriada mejora el resultado perinatal en mujeres con RPPM precoz (3). Este meta-análisis incluye solo estudios observacionales y no estudios randomizados controlados. Solo existen 2 estudios randominazos controlados que reclutan pacientes con RPPM entre 24 y 34 semanas de gestación. No existe evidencia de estudios randomizados controlados que soporten el uso de amnioinfusión en pacientes con RPPM durante las semanas críticas del desarrollo que son entre las 16 y 24 semanas de gestación.

\section{B. El estudio (1)}

Diseño: Estudio clínico randomizado, multicéntrico, controlado, no ciego, realizado en el periodo comprendido entre el 2002 y el 2009 en cuatro hospitales del Reino Unido. Pacientes: 56 pacientes con RPPM entre las semanas $16+0$ y 24+0 de gestación y con bolsillo mayor de líquido amniótico menor a $2 \mathrm{~cm}$. Intervención: En 28 pacientes se realizó amnioinfusión seriada semanal si el bolsillo mayor de líquido amniótico medido cada siete días era $<2 \mathrm{~cm}$ hasta la semana 37. Comparación: En 28 pacientes se realizó un manejo expectante hasta las 37 semanas. Resultado primario: Mortalidad perinatal, morbilidad materna y neonatal precoz. Resultado secundario: Morbilidad respiratoria y neurodesarrollo a largo plazo. Resultados: No se demostraron diferencias en los resultados primarios del estudio. No fue posible demostrar diferencias estadísticamente significativas en los resultados secundarios producto del pequeño número de pacientes sobrevivientes a largo plazo.

\section{Análisis crítico}

Validez interna: Estudio con descripción apropiada de los criterios de inclusión y exclusión, randomizado, con ocultamiento de la secuencia, similitud de pacientes en ambos grupos, con seguimiento completo y análisis por intención de tratar. El tamaño muestra fue calculado considerando un resultado compuesto (mortalidad y morbilidad respiratoria en los sobrevivientes), sin embargo, finalmente no se trató de completar ese tamaño muestral, y considerar el estudio solo como un piloto y reclutar pacientes hasta el término del financiamiento. La tasa de mortalidad perinatal fue mayor de lo esperado y la proporción de sobrevivientes sanos es mucho 
menor de lo previsto, reduciendo críticamente el poder del estudio. No se encontraron diferencias estadísticamente significativas en ninguno de los resultados entre los dos grupos; sin embargo, el número de pacientes fue demasiado pequeño para obtener conclusiones definitivas respecto del resultado primario. Este estudio no fue diseñado para mostrar diferencias significativas de los resultados secundarios (morbilidad respiratoria y alteraciones del neurodesarrollo a largo plazo). Otro aspecto deficiente es la falta de ciego tanto de los clínicos como de los participantes; no está descrito en la publicación si los recolectores de datos o asignadores de resultados fueron ciegos a la intervención. Validez externa: El estudio podría ser aplicable a nuestra población, ya que las medidas y cuidados descritos para este tipo de pacientes es similar a la nuestra. La conducta de realizar amnioinfusión seriada es relativamente de bajo costo y cualquier unidad de Medicina Materno Fetal de nuestro país cuenta con el personal capacitado para la realización de esta intervención. Conclusiones: Estudio de buena calidad (randomizado), sin embargo, con importantes fuentes potenciales de sesgo: número de pacientes reducidos, sin respeto por el cálculo de tamaño muestral y la falta de ciego en todos los niveles. Los resultados de esta investigación no resultan de utilidad para cambiar nuestra práctica clínica habitual y no deben ser considerados hasta nuevos estudios clínicos randomizados con un número mayor de pacientes.

\section{REFERENCIAS}

1. Roberts D, Vause S, Martin W, Green P, Walkinshaw S, Bricker L, Beardsmore C, Shaw N, McKay A, Skotny G, Williamson P, Alfirevic Z. Amnioinfusion in very early preterm premature rupture of membranes - pregnancy, neonatal and maternal outcomes in the AMIPROM randomised controlled pilot study. Ultrasound Obstet Gynecol 2013. doi: 10.1002/uog.13258. [Epub ahead of print]

2. Locatelli A, Ghidini A, Verderio M, Andreani M, Strobelt $N$, Pezzullo J, Vergani P. Predictors of perinatal survival in a cohort of pregnancies with severe oligohydramnios due to premature rupture of membranes at $<26$ weeks managed with serial amnioinfusions. Eur J Obstet Gynecol Reprod Biol 2006;128(1-2):97102.

3. Porat S, Amsalem H, Shah PS, Murphy KE. Transabdominal amnioinfusion for preterm premature rupture of membranes: asystematic review and metaanalysis of randomized and observational studies. Am J Obstet Gynecol 2012;207(5):393.e1-11. 